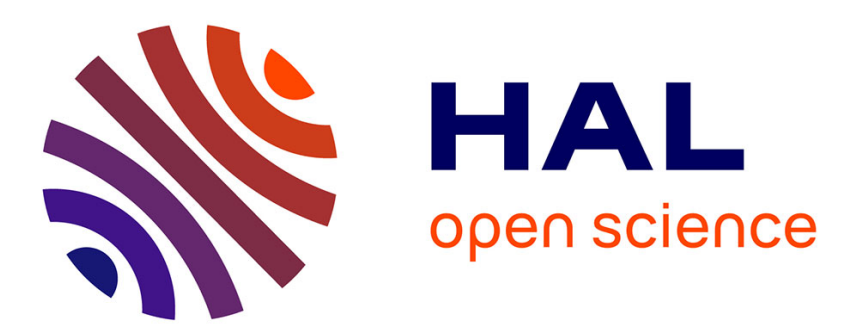

\title{
Effect of anomalous diffusion of fluctuating Cooper pairs on the density of states of superconducting $\mathrm{NbN}$ thin films
}

\author{
Pietro Brighi, Marco Grilli, Brigitte C. Leridon, Sergio Caprara
}

\section{- To cite this version:}

Pietro Brighi, Marco Grilli, Brigitte C. Leridon, Sergio Caprara. Effect of anomalous diffusion of fluctuating Cooper pairs on the density of states of superconducting $\mathrm{NbN}$ thin films. Physical Review B: Condensed Matter (1978-1997), 2019, 100, pp.174518. 10.1103/PhysRevB.100.174518 . hal03024481

\section{HAL Id: hal-03024481 \\ https://hal.science/hal-03024481}

Submitted on 25 Nov 2020

HAL is a multi-disciplinary open access archive for the deposit and dissemination of scientific research documents, whether they are published or not. The documents may come from teaching and research institutions in France or abroad, or from public or private research centers.
L'archive ouverte pluridisciplinaire HAL, est destinée au dépôt et à la diffusion de documents scientifiques de niveau recherche, publiés ou non, émanant des établissements d'enseignement et de recherche français ou étrangers, des laboratoires publics ou privés. 


\title{
Effect of anomalous diffusion of fluctuating Cooper pairs on the density of states of superconducting $\mathrm{NbN}$ thin films
}

\author{
Pietro Brighi $\odot,{ }^{1}$ Marco Grilli $\odot,{ }^{2,3, *}$ Brigitte Leridon $\odot,{ }^{4}$ and Sergio Caprara $\odot^{2,3}$ \\ ${ }^{1}$ IST Austria, Am Campus 1, 3400, Klosterneuburg, Austria \\ ${ }^{2}$ ISC-CNR, via dei Taurini 19, I-00185 Rome, Italy \\ ${ }^{3}$ Department of Physics, Università La Sapienza, Piazzale A. Moro 5, I-00185 Rome, Italy \\ ${ }^{4}$ LPEM, ESPCI Paris, CNRS, Université PSL, Sorbonne Universités, 10 rue Vauquelin, 75005 Paris, France
}

(Received 31 July 2019; revised manuscript received 3 October 2019; published 25 November 2019)

\begin{abstract}
Recent scanning tunneling microscopy experiments in $\mathrm{NbN}$ thin disordered superconducting films found an emergent inhomogeneity at the scale of tens of nanometers. This inhomogeneity is mirrored by an apparent dimensional crossover in the paraconductivity measured in transport above the superconducting critical temperature $T_{c}$. This behavior was interpreted in terms of an anomalous diffusion of fluctuating Cooper pairs that display a quasiconfinement (i.e., a slowing down of their diffusive dynamics) on length scales shorter than the inhomogeneity identified by tunneling experiments. Here, we assume this anomalous diffusive behavior of fluctuating Cooper pairs and calculate the effect of these fluctuations on the electron density of states above $T_{c}$. We find that the density of states is substantially suppressed up to temperatures well above $T_{c}$. This behavior, which is closely reminiscent of a pseudogap, only arises from the anomalous diffusion of fluctuating Cooper pairs in the absence of stable preformed pairs, setting the stage for an intermediate behavior between the two common paradigms in the superconducting-insulator transition, namely, the localization of Cooper pairs (the so-called bosonic scenario) and the breaking of Cooper pairs into unpaired electrons due to strong disorder (the so-called fermionic scenario).
\end{abstract}

DOI: 10.1103/PhysRevB.100.174518

\section{INTRODUCTION}

The physics of the superconductor-insulator transition (SIT) in disordered superconducting thin films [1] is attracting an ever increasing interest both for applicative purposes [2,3] and for fundamental reasons [4-6]. In this latter regard, while in structurally granular thin films the intrinsic granularity plays an evident role, the situation is more involved in nominally homogeneous (i.e., nongranular) disordered thin films. On the one hand, what is often referred to as the "fermionic" scenario proposes that the SIT is driven by the reduced screening of the Coulomb repulsion with increasing disorder, that leads to a weakening of pairing and to a reduction of the critical temperature $T_{c}$ [7]. In this case, the insulating state hosts localized fermions and standard paraconductive fluctuations are expected above $T_{c}$, due to Gaussiandistributed short-lived Cooper pairs. In granular systems, the situation may be more complex with the appearance of various crossovers theoretically predicted [8] and experimentally observed [9].

On the other hand, tightly bound Cooper pairs survive the SIT in a "bosonic" scenario, in which the gap persists above $T_{c}$ despite the loss of phase coherence. In this framework, the bosonic pairs either localize because the disorder-enhanced Coulomb interaction destroys their phase-coherent motion at large scales $[10,11]$, or disorder itself blurs the phase

*Corresponding author: marco.grilli@roma1.infn.it coherence without any relevant role of the Coulomb repulsion [12-15]. In the latter case, it was also proposed that the superconducting state is characterized by an emergent disordered glassy phase [15], with filamentary superconducting currents [12]. An anomalous distribution of the superconducting order parameter was proposed by theorists $[15,16]$, and observed experimentally $[6,17]$. A numerical approach to uniformly disordered superconductors [18] has also shown that there is a continuous evolution [19] from the weak-disorder limit, where the system has a rather homogeneous fermionic character, to the strong-disorder limit, where marked inhomogeneities appear in the superconducting order parameter, with an emergent bosonic nature characterized by a single-particle gap persisting on the insulating side of the SIT. A great deal of experimental activity has been devoted to this more disordered realization of the SIT [6,20]. The intermediate situation, where Cooper pairs begin to evolve into bosonic pairs, but keep their fermionic character, has been recently investigated by scanning tunneling microscopy (STM) and transport measurements in $\mathrm{NbN}$ thin films. STM revealed the occurrence of an emergent inhomogeneous state and pseudogap effects over scales of a few tens of nanometers [21]. This intermediatescale inhomogeneity affected the transport properties with the paraconductivity displaying a crossover from a seemingly zero-dimensional $[22,23]$ Aslasmazov-Larkin (AL) behavior to the expected two-dimensional behavior when $T_{c}$ was approached from above. This crossover was interpreted in terms of an anomalous slowing down of the dynamics of fluctuating Cooper pairs at length scales smaller than those set by the 

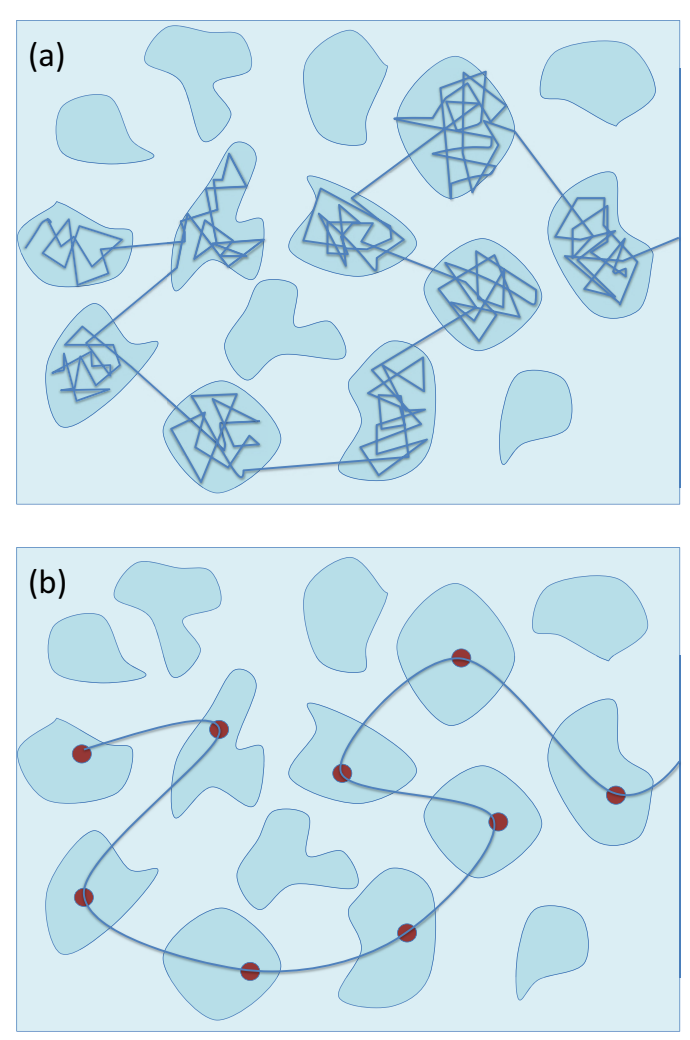

FIG. 1. (Adapted form Fig. 7 of Ref. [21]) Sketch of the anomalous diffusion of the fluctuating Cooper pairs induced by the emergent inhomogeneity in $\mathrm{NbN}$ thin films. (a) The fluctuating Cooper pairs above $T_{c}$ diffuse in the system, but are slowed inside the emergent inhomogeneities (represented by the darker regions). The multiple scattering inside these regions pictorially represents the diffusion slowdown leading to a quasiconfinement of the fluctuating Cooper pairs. This mechanism is expected to give rise to a pseudogap and to the zero-dimensional character of the paraconductive fluctuations. (b) Over longer distance and timescales a "coarsegrained" standard diffusive behavior of the fluctuating Cooper pairs is recovered, leading to the usual two-dimensional AL behavior of the paraconductivity.

emergent inhomogeneity. Figure 1 pictorially illustrates this effect. The theoretical framework elaborated to explain the experiments in Ref. [21] and the analysis reported in this work provides therefore a third paradigm "intermediate" between the fermionic and the bosonic ones. In particular, in this paper we explore other consequences of this intriguing emergence of inhomogeneity. Specifically, we consider the effects of this anomalous dynamics of the fluctuating Cooper pairs on the electron density of states above $T_{c}$, showing that, in the presence of disorder, the slowing down of Cooper pair fluctuations may give rise to pseudogap effects on the same length scale over which the Cooper pairs are quasiconfined. This will explain the occurrence of the pseudogap observed in STM experiments despite the persistence of purely fluctuating Cooper pairs (as opposed to the stable pairs of the bosonic scenario), which are needed to account for the standard Aslamazov-Larkin paraconductivity.

The structure of this paper is as follows. In Sec. II we present our phenomenological scheme involving the anomalous diffusion of fluctuating Cooper pairs. Section III is devoted to the theoretical many-body calculation that determines the effects of the fluctuating Cooper pairs on the electron density of states (DOS). This section will also present the systematic analysis of these effects and a comparison with the experimental results of STM on NbN. Section IV contains our final remarks and conclusions.

\section{THEORETICAL BACKGROUND}

The core idea of the phenomenological theoretical framework used in Ref. [21] to fit the paraconductivity of $\mathrm{NbN}$ thin film is that there exist some regions, inside the superconducting film, where the lifetime of fluctuating Cooper pairs above the superconducting critical temperature $T_{c}$ is longer than expected for a standard diffusion process, resulting in a quasiconfinement of the fluctuating Cooper pairs within nanoscopic regions, dubbed supergrains, to emphasize the fact that they do not correspond to the structural grains. Indeed, these regions, of a linear dimension $L_{i} \approx 50 \mathrm{~nm}$, are much larger than the typical disorder length scale of a few nanometers structurally present in nominally homogeneous NbN films. In this scenario, fluctuations with characteristic wavelength smaller than the typical inhomogeneity dimension $L_{i}$ are more long lived than they would be in a standard diffusion process, thus giving an explanation to the experimentally observed paraconductivity anomalies, resembling a zero-dimensional behavior.

It is well established from the theory of fluctuations in superconductors, that the propagator of the fluctuating Cooper pairs in the weak-coupling (BCS) limit has the following form [22,23]:

$$
L(\mathbf{q}, \Omega) \simeq-\frac{8 T}{N_{0}} \frac{1}{\tau_{\mathrm{GL}}^{-1}+\varepsilon(\mathbf{q})-i \Omega},
$$

where $N_{0}$ is the electron DOS at the Fermi level, $\tau_{\mathrm{GL}}^{-1}=$ $\frac{8 T_{c}}{\pi} \log \frac{T}{T_{c}}$ is the inverse Ginzburg-Landau lifetime, and $\varepsilon(\mathbf{q})$ is the dispersion law, determining the increase of the inverse diffusion time with increasing magnitude of the wave vector $\mathbf{q}$ of the fluctuating mode. In this work we adopt units such that the Planck constant $\hbar$ and the Boltzmann constant $k_{B}$ are unity.

The standard results from fluctuation theory are obtained with a quadratic diffusion law $\varepsilon(\mathbf{q})=D q^{2}$ (where $q \equiv|\mathbf{q}|$ ). On the other hand, the anomalous diffusion should give higher lifetimes for wavelengths smaller than $L_{i}$ and recover the standard behavior at larger scales. In particular, it was found [see the inset in Fig. 4(b) of Ref. [21]] that the pararesistivity (i.e., the inverse of the paraconductivity) crosses over from a seemingly zero-dimensional (0D) behavior $\propto\left(T-\tilde{T}_{c}\right)^{2}$ to the expected two-dimensional (2D) behavior $\propto\left(T-T_{c}\right)$, with $\tilde{T}_{c}$ being somewhat smaller than $T_{c}$. A good description of the 0D-2D crossover occurring in the experimental paraconductivity measurements on $\mathrm{NbN}$ thin films was achieved through the following anomalous diffusive expression:

$$
\varepsilon(\mathbf{q})=D \bar{q}^{2} \log \left(1+\frac{q^{2}}{\bar{q}^{2}}\right)
$$




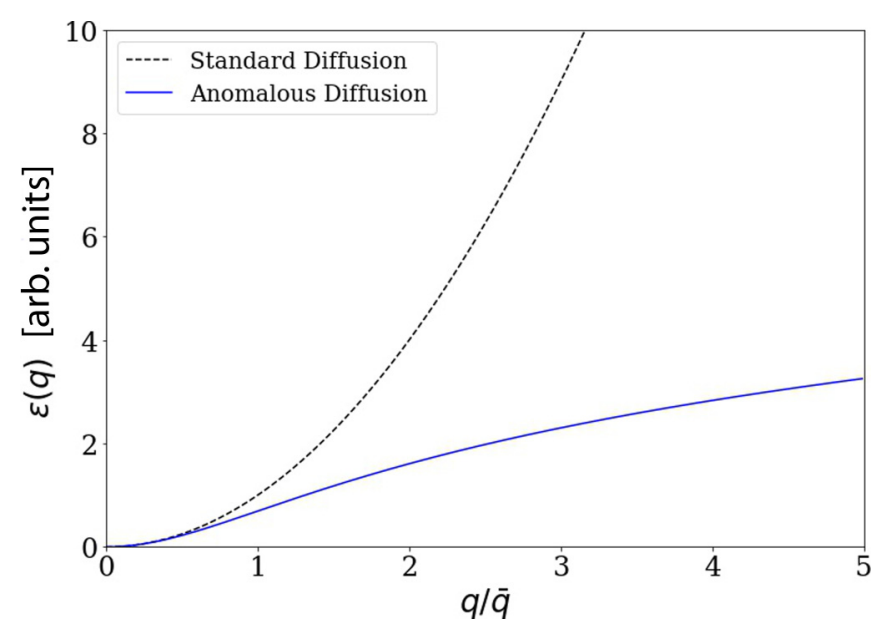

FIG. 2. The two different diffusion laws: the dashed line represents the standard quadratic law, while the blue curve is the anomalous one. It is possible to observe that on small momenta the two coincide asymptotically, while above $q / \bar{q}=1$ the anomalous diffusion becomes quite smaller than the standard one, representing the quasiconfinement of the fluctuating Cooper pairs inside the supergrains.

where $D$ is the diffusion constant and $\bar{q} \approx L_{i}^{-1}$. The theoretical framework leading to the above expression is discussed in Appendices A, B, and C. Here, we notice that the experiments only constrain the asymptotic behaviors for $q \ll \bar{q}$ or $q \gg \bar{q}$, while the intermediate behavior, as it customarily occurs in crossover phenomena, is much less constrained and rather immaterial. Figure 2 compares the standard quadratic diffusion law (black dashed line) with the anomalous diffusion of Eq. (2) (solid blue line). We point out that the value of the diffusion constant $D$ in the presence of disorder may be severely suppressed with respect to the standard BCS value [22,23]. As long as $D$ stays finite, however, its value does not appear in the AL paraconductivity in two dimensions, as the consequence of a cancellation enforced by gauge invariance [24]. Disorder may also introduce corrections to the BCS value of $\tau_{\mathrm{GL}}^{-1}$, as it seems indeed to be the case in the more strongly disordered $\mathrm{NbN}$ films [21].

At small momenta the two lines coincide since the system is normally diffusive over large length scales. At $q \geqslant \bar{q}$ instead, the anomalous diffusion becomes much smaller than the quadratic one, indicating that for these modes the diffusion occurs with characteristic frequencies $\Omega \sim \tau_{\mathrm{GL}}^{-1}+\varepsilon(\mathbf{q})$ much smaller (i.e., with much longer characteristic times) than in the standard case $\Omega \sim \tau_{\mathrm{GL}}^{-1}+D q^{2}$

This work aims at understanding the effects of such an anomalous diffusion of fluctuating Cooper pairs on the electron density of states of a two-dimensional superconductor.

\section{DENSITY OF STATES FROM ANOMALOUS DIFFUSION OF FLUCTUATING COOPER PAIRS}

Following a standard quantum many-body approach, the density of states of an electron system is given by the imaginary part of its retarded Green function $\mathcal{G}^{R}(\mathbf{k}, \omega)$ :

$$
N(\omega)=-\frac{1}{2 \pi} \int \frac{d^{2} \mathbf{k}}{(2 \pi)^{2}} \operatorname{Im} m\left[\mathcal{G}^{R}(\mathbf{k}, \omega)\right] .
$$

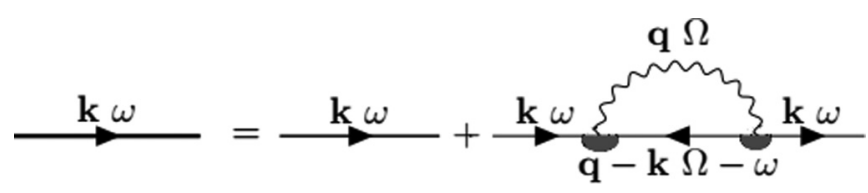

FIG. 3. Feynman diagram for the contribution of fluctuating Cooper pairs to the electron Green function. The thick (thin) solid line represents the dressed (bare) electron Green function, the wavy line represents the propagator of fluctuating Cooper pair, and the shaded semicircles represent the impurity ladders that dress the electron-Cooper pair fluctuation vertex and embody the effect of microscopic disorder.

The Feynman diagrams of Fig. 3 give the fluctuative contribution to the Green function in the dirty limit [25-27]. The wavy line corresponds to the propagator of fluctuating Cooper pairs $L\left(\mathbf{q}, \Omega_{n}\right)$. While here we adopt the finite-temperature formalism with Matsubara frequencies $\Omega_{n}$ [22,23], the form in Eq. (1) has been analytically continued to real frequency $\Omega$ in order to obtain a diffusive pole for the fluctuating Cooper pairs above $T_{c}$. The shaded semicircles are vertices coupling the Cooper pair fluctuations with the electron quasiparticles. In the presence of disorder due to quenched impurities and in the so-called ladder approximation [22,23] they read as

$$
\Lambda\left(\mathbf{q}, \omega_{m}, \Omega_{n}\right)=\frac{1}{\tau} \frac{1}{\varepsilon(\mathbf{q})+\left|2 \omega_{m}+\frac{\operatorname{sign}\left(\omega_{m}\right)}{\tau}-\Omega_{n}\right|},
$$

where $\tau$ is the relaxation time of the electronic scattering on the impurities, while $\omega_{m}=(2 m+1) \pi T$ and $\Omega_{n}=2 n \pi T$ are the fermion and boson Matsubara frequencies, respectively.

From the second diagram in the right-hand side of Fig. 3, we obtain the corrections to the electron Green function due to fluctuating Cooper pairs. Upon integration over the electron momenta $\mathbf{k}$, we obtain

$$
\begin{aligned}
\delta G\left(\omega_{m}\right)= & -\frac{T}{\pi N_{0}}\left[\sum_{\Omega_{n}} \int \frac{d^{2} \mathbf{q}}{(2 \pi)^{2}} L\left(\mathbf{q}, i\left|\Omega_{n}\right|\right) \Lambda^{2}\left(\mathbf{q}, \omega_{m}, \Omega_{n}\right)\right. \\
& \left.\times \int \frac{d^{2} \mathbf{k}}{(2 \pi)^{2}} G_{0}^{2}\left(\mathbf{k}, \omega_{m}\right) G_{0}\left(\mathbf{q}-\mathbf{k}, \Omega_{n}-\omega_{m}\right)\right]
\end{aligned}
$$

where $G_{0}\left(\mathbf{k}, \omega_{m}\right)=\left[i \omega_{m}-v_{F}\left(k-k_{F}\right)\right]^{-1}$ is the free-electron Green function in the Matsubara formalism, $v_{F}$ and $k_{F}$ being the Fermi velocity and Fermi wave vector, respectively. The sum over boson Matsubara frequencies and the integral over fermion momenta can be carried out analytically as it was done in Ref. [26] for the case of standard diffusion of fluctuating Cooper pairs. Analytically continuing the fermion frequencies $\left(i \omega_{m} \rightarrow \omega-i 0^{+}\right)$and taking the imaginary part in Eq. (5), one obtains the variation of the DOS:

$$
\begin{aligned}
\delta N(\omega)= & -\frac{16 T^{2}}{\pi N_{0} \tau} \int \frac{q d q}{\left[\tau_{\mathrm{GL}}^{-1}+\varepsilon(q)\right]\left[\tau_{\mathrm{GL}}^{-1}+2 \varepsilon(q)\right]^{2}} \\
& \times \frac{\left(4|\omega|+\frac{1}{2 \tau}\right)^{2}-\left[\tau_{\mathrm{GL}}^{-1}+\varepsilon(q)\right]^{2}}{\left\{\left(4|\omega|+\frac{1}{2 \tau}\right)^{2}+\left[\tau_{\mathrm{GL}}^{-1}+\varepsilon(q)\right]^{2}\right\}^{2}},
\end{aligned}
$$




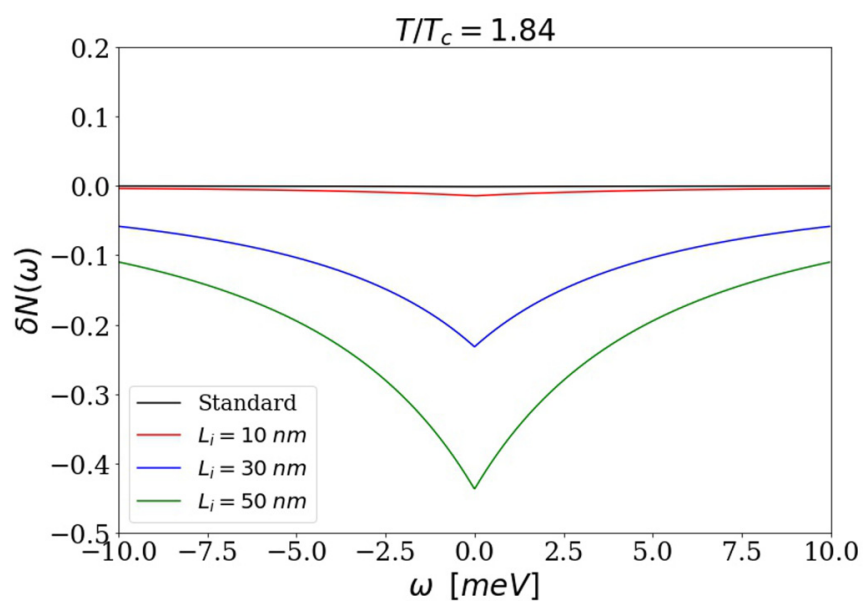

FIG. 4. Effects of (anomalously diffusing) Cooper pair fluctuations on the quasiparticle DOS at $T / T_{C}=1.84$, at fixed level of disorder. The elastic scattering rate is $\frac{1}{\tau}=20 \mathrm{meV} \approx 0.067 E_{F}$, where $E_{F}=v_{F} k_{F}$ is the Fermi energy. The black line shows the result for standard diffusion, with barely any visible effect as compared to the much stronger suppression observed in the case of anomalous diffusion.

where the last integration over $q=|\mathbf{q}|$ can be carried out numerically, and we made explicit that $\varepsilon(\mathbf{q})$ only depends on $q$.

Starting from the numerical evaluation of Eq. (6), we systematically investigate the role of disorder (namely, of the scattering time $\tau$ ) and of the inhomogeneity length scale $\left(L_{i}\right)$. One first generic finding is that the anomalous diffusion of fluctuating Cooper pairs substantially increases the size and the extension in temperature above $T_{c}$ of the pseudogap effects (i.e., of the partial DOS suppression that occurs above $T_{c}$ because of superconducting fluctuations) in comparison with the standard diffusion law $\varepsilon(q)=D q^{2}$. These effects are markedly larger in the disordered case than in the clean case (i.e., in the $\tau \rightarrow \infty$ limit): only in the disordered case the DOS suppression can become quantitatively comparable to the suppression observed by STM experiments in NbN. This is why in the following we will focus on the disordered case only.

In Fig. 4, different $\delta N(\omega)$ curves are compared, at $T / T_{c}=$ 1.84 (corresponding to $T=7 \mathrm{~K}$ for the sample $X_{0}$ in Ref. [21], having $T_{c}=3.8 \mathrm{~K}$ ), rather far above $T_{c}$. The black line represents the curve obtained with the standard diffusion law, while the colored ones have been obtained with different values of $L_{i}=20,30,50 \mathrm{~nm}$ [i.e., different $\bar{q}$ in Eq. (2)], all in the range of the inhomogeneity sizes observed in Ref. [21].

It is evident that anomalous diffusion greatly enhances the pseudogap effects. Quite far above $T_{c}$, at $T / T_{c}=1.84$, the DOS suppression in the standard diffusion case (black solid line) is barely appreciable adopting a common scale, being much smaller than the suppression observed in the case of anomalous diffusion. This demonstrates that these pseudogap effects are quite robust in temperature. We also notice that increasing the parameter $L_{i}$, we obtain a more pronounced DOS suppression, as this reduces $\bar{q}$, thus extending the region in $q$ space where the anomalous diffusion of fluctuating Cooper pairs occurs.

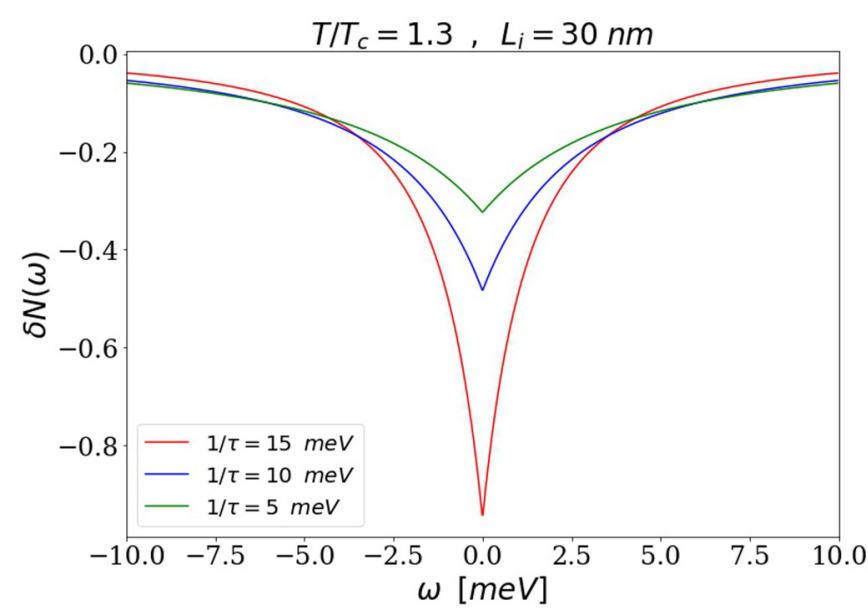

FIG. 5. Effect of the disorder strength on the Cooper fluctuation contribution to the DOS at $T / T_{c}=1.3$ and $L_{i}=30 \mathrm{~nm}$.

In Fig. 5, we report the results of our analysis on the effects of disorder. It is evident that, for a given fixed value of $L_{i}=30 \mathrm{~nm}$, at $T / T_{c}=1.3$, the DOS suppression increases by increasing the elastic scattering rate $\frac{1}{\tau}$. This indicates that the homogeneously distributed microscopic disorder represented by the impurities is cooperative with the large-scale inhomogeneity producing a stronger effect of the anomalous diffusion of Cooper pair fluctuations.

The results reported above show that the pseudogap effects induced by the anomalous diffusion of fluctuating Cooper pairs are large enough to account for the sizable pseudogap effects observed by STM in NbN. On the other hand, the DOS corrections shown in Figs. 4 and 5 systematically display a suppression (i.e., a negative correction) with respect to the reference DOS of the normal metallic state at high temperature. Therefore, results cannot account for the experimental observation of coherence effects that rather symmetrically produce an enhancement of DOS at finite energy (finite bias in tunnel experiments) above or below the Fermi level $(\omega=0)$. Of course, the spectral weight is not lost in our calculations, but is simply distributed over a very broad range, larger than the range of the figures. To obtain more realistic tunnel spectra, it is crucial to recall that the two different fluctuational regimes observed in the transport experiments of Ref. [21], appear to be characterized by two different critical temperatures. In other words, the transition temperature in the two-dimensional regime that sets in when the true $T_{c}$ is approached, is larger than the critical temperature of the anomalously diffusing regime. This latter is when fluctuating Cooper pairs that shape the paraconductivity data at higher temperatures further away from $T_{c}$ display the seemingly zero-dimensional character. This observation, substantiated in Fig. 4(b) of Ref. [21], is not surprising because the $0 \mathrm{D}$ regime occurs at higher temperatures and is dominated by the anomalous diffusion of fluctuating Cooper pairs over length scales shorter than $L_{i}$ and at these scales the system is seemingly unaware of the actual critical temperature $T_{c}$ at which the true two-dimensional global superconducting state is established [28].

Therefore, in order to mimic this "flowing" of the critical temperature when shorter and shorter length scales (i.e., larger and larger inverse timescales) dominate the Cooper 


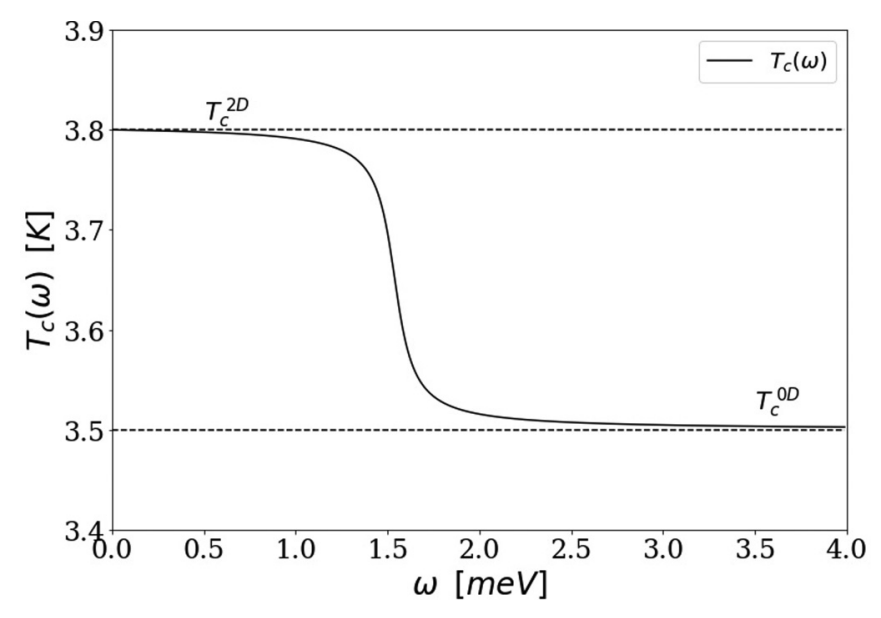

FIG. 6. The curve representing the proposed $T_{c}(\omega)$. A smooth change from the two-dimensional (2D) regime to the zerodimensional (0D) one can be tuned using an arctangent interpolating function. The values of the asymptotic critical temperatures are those obtained in Ref. [21].

fluctuations, we assumed a smooth steplike energy dependent $T_{c}(\omega)$, as shown in Fig. 6. Here, $T_{c}^{0 \mathrm{D}}$ and $T_{c}^{2 \mathrm{D}}$ are taken from the already mentioned pararesistivity measurements [see the inset in Fig. 4(b) of Ref. [21]]. In particular, for $T_{c}(\omega)$ reported in Fig. 6 we used the values found for sample $X_{0}$ of Ref. [21] (see also the Supplemental Material of this reference).

When evaluating Eq. (6), the $T_{c}$ appearing in the definition of $\tau_{\mathrm{GL}}$ is replaced by $T_{c}(\omega)$. With this phenomenological assumption, we then obtain the fitting of tunnel spectrum reported in Fig. 7 for $T=1.1 T_{c}$ (corresponding to the experimental conditions for sample $X_{0}$ in Fig. 2 of Ref. [21]). The data were obtained taking the experimental spectra of the

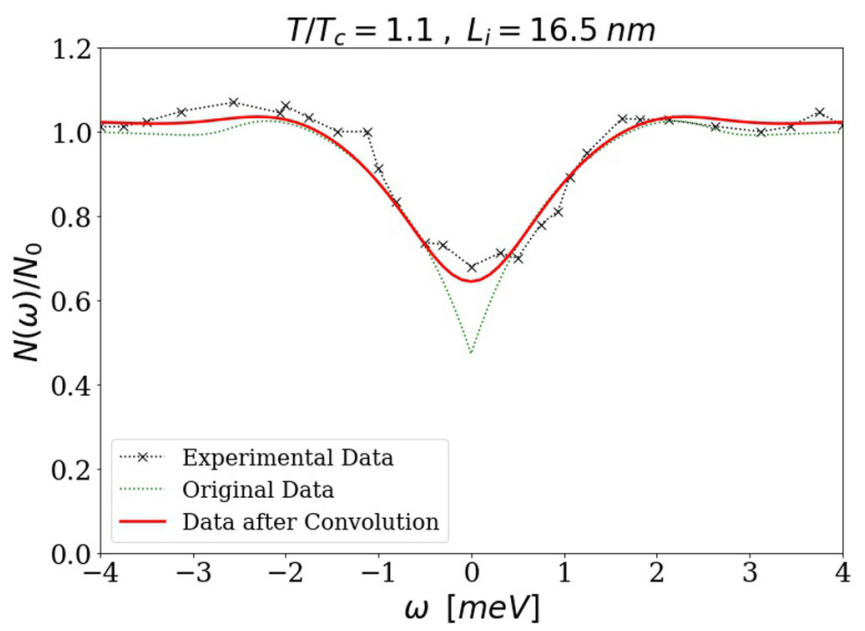

FIG. 7. Comparison of the theoretical curve (red line) for $N(\omega) / N_{0}$, where $N(\omega)=N_{0}+\delta N(\omega)$ [see Eq. (6)], with experimental data $(\times)$ at a temperature $T=1.1 T_{c}$ and with $L_{i}=16.5 \mathrm{~nm}$ as typical size of the inhomogeneity. Here, $1 / \tau=5 \mathrm{meV}$. The theoretical curve has been convoluted with a Gaussian with variance $\sigma=$ $0.4 \mathrm{meV}$ to account for the experimental resolution. The theoretical curve before convolution is also reported with green dotted line for comparison. large pseudogap regions (the supergrains) and dividing them by the corresponding measurements in the small pseudogap regions [the red and blue curves, respectively, in Fig. 2(b) of Ref. [21]], to extract the contribution of anomalously diffusing Cooper pair fluctuations and get rid of any background contribution. We used $L_{i}$ and $\tau$ as adjustable parameters. The fitting DOS suppression (normalized to the DOS in the metallic state) was obtained for $L_{i}=16.5 \mathrm{~nm}$, quite comparable with the typical size of supergrains observed in $\mathrm{NbN}$ films, and for $1 / \tau=5 \mathrm{meV}$, for which we have no independent determination. Figure 7 reports the comparison between the theoretical calculations and the experimental data. The theoretical curve has also been convoluted with a Gaussian with variance $\sigma=$ $0.4 \mathrm{meV}$ to account for the experimental resolution.

The agreement between the theoretical curve and experimental data is quite satisfactory at every $\omega$. The convolution with the finite-resolution Gaussian plays a role only near $\omega=0$, where it transforms the cusplike theoretical curves (see, e.g., Fig. 5) in the more rounded red line of Fig. 7.

\section{CONCLUSIONS AND OUTLOOKS}

In this paper, we presented a theory for the DOS suppression due to anomalously diffusing fluctuating Cooper pairs above the critical temperature $T_{c}$ of a two-dimensional superconductor. Our theoretical results highlight the effectiveness of the anomalous diffusion of fluctuating Cooper pairs in enhancing the pseudogap at temperatures well above $T_{c}$. Since a similar effect was observed experimentally in some ultrathin NbN films, we compared our curves with STM experiments, in order to get an estimate of the parameters of the theory. Good agreement with experimental data is achieved, especially after introducing the natural idea that Cooper fluctuations at different energies may "perceive" different critical temperatures. This led us to the phenomenological assumption that the critical temperature should, in our theory, be frequency dependent, as a consequence of the 0D-2D crossover, with the asymptotic high-frequency (0D) and low-frequency (2D) values fitted to match the experimental paraconductivity [21], and a smooth interpolating behavior at intermediate frequency. This work has been carried out on a phenomenological basis in the form of the anomalous diffusive law of Cooper pairs and of $T_{c}(\omega)$ and the understanding of the actual microscopical reasons giving rise to the inhomogeneities, their length scales, and their effects on the above laws is lacking. Nevertheless, we point out that our work opens the way to a different microscopic understanding of the SIT, in which Cooper pairs are not stable bosonic entities, but keep a fluctuating character. It is their slowing down that produces marked pseudogap effects mimicking that of preformed pairs and strengthening the idea of a gradual and continuous evolution between the bosonic and fermionic scenarios.

\section{ACKNOWLEDGMENTS}

S.C. thanks all the colleagues of the ESPCI in Paris for their warm hospitality and for many useful discussion while part of this work was done. P.B. acknowledges fruitful discussions with Professor L. G. A. Molinari. S.C. and M.G. acknowledge financial support of 
the Sapienza Università di Roma under the Ateneo 2017 (Grant No. RM11715C642E8370) and Ateneo 2018 (Grant No. RM11816431DBA5AF projects. Part of the work was supported through the Chaire Joliot at École Supérieure de Physique et de Chimie Industrielles de la Ville de Paris (ESPCI). This work was also supported by EU through the COST action CA16218 NanocoHybri.

\section{APPENDIX A: ZERO-DIMENSIONAL AL FLUCTUATIONS}

The theory discussed here was previously elaborated to explain the experimental paraconductivity measurements reported in Ref. [21]. Owing to the more theoretical character of this work, we feel it useful to provide in these appendices a more detailed description of this theoretical framework.

To discuss AL paraconductivity, we shall assume overall isotropy (at least in the average) and start from the expression [29]

$$
\delta \sigma_{D}(\varepsilon)=\frac{\pi e^{2}}{4 \hbar} \int \frac{d^{D} \mathbf{q}}{(2 \pi)^{D}} \frac{1}{D}\left(\nabla_{\mathbf{q}} v_{\mathbf{q}}\right)^{2} \frac{1}{\left(\varepsilon+v_{\mathbf{q}}\right)^{3}},
$$

that reproduces the standard $\mathrm{AL}$ result in $D$ dimensions with $v_{\mathbf{q}} \equiv \xi_{0}^{2}|\mathbf{q}|^{2}$, where $\xi_{0}$ is the coherence length and $\varepsilon \equiv$ $\log \left(T / T_{c}\right), T_{c}$ being the critical temperature. In these appendices we use the more convenient dimensionless quantities $\varepsilon$ and $v_{\mathbf{q}}$, which are related to the dimensional quantities used in the main text by the following relations: $\tau_{\mathrm{GL}}^{-1}=8 T_{c} \varepsilon / \pi$ and $\varepsilon(\mathbf{q})=8 T_{c} v_{\mathbf{q}} / \pi$. The expression in Eq. (A1) appears to be rather general [24], although the precise expression of $\varepsilon$ may change depending on the microscopic theory giving rise to collective paraconductive fluctuations, so that the expression $\varepsilon \equiv \alpha\left(T-T_{c}\right) / T_{c}$, with a dimensionless prefactor $\alpha$ not necessarily equal to one, may be used.

In this Appendix, we shall study the formal limit $D \rightarrow$ 0 of the standard AL paraconductivity, while in the next appendices we shall study more general expressions, apt to describe the phenomenology of $\mathrm{NbN}$ thin films. The angular integration in Eq. (A1) factors out and, observing that $v_{\mathbf{q}}$ only depends on $q \equiv|\mathbf{q}|$, one obtains

$$
\delta \sigma_{D}=\frac{\pi e^{2} \Omega_{D}}{4 \hbar D(2 \pi)^{D}} \int d q q^{D-1}\left(\frac{d v_{q}}{d q}\right)^{2} \frac{1}{\left(\varepsilon+v_{q}\right)^{3}},
$$

where

$$
\Omega_{D}=\frac{2 \pi^{D / 2}}{\Gamma\left(\frac{D}{2}\right)}
$$

is the surface of the unitary sphere in $D$ dimensions and $\Gamma(z)$ is the Euler gamma function. Exploiting the expression of $v_{q}$ one obtains

$$
\delta \sigma_{D}=\frac{\pi e^{2} \Omega_{D} \xi_{0}^{4}}{\hbar D(2 \pi)^{D}} \int_{0}^{\infty} d q \frac{q^{D+1}}{\left(\varepsilon+\xi_{0}^{2} q^{2}\right)^{3}} .
$$

When $D \rightarrow 0, \Omega_{D}$ inherits the singularity of $\Gamma(D / 2) \approx 2 / D$, so that $\Omega_{D} \approx D$. Then,

$$
\begin{aligned}
\delta \sigma_{D \rightarrow 0} & =\frac{\pi e^{2} \xi_{0}^{4}}{\hbar} \int_{0}^{\infty} d q \frac{q}{\left(\varepsilon+\xi_{0}^{2} q^{2}\right)^{3}} \\
& =\frac{\pi e^{2} \xi_{0}^{2}}{2 \hbar} \int_{0}^{\infty} d z \frac{1}{(\varepsilon+z)^{3}},
\end{aligned}
$$

with the change of variable $z=\xi_{0}^{2} q^{2}$. The integral over $z$ is straightforward and one obtains

$$
\delta \sigma_{D \rightarrow 0}=\frac{\pi e^{2} \xi_{0}^{2}}{4 \hbar \varepsilon^{2}} .
$$

The above result shows that there exists a well-defined limit of the AL paraconductivity as $D \rightarrow 0$, that scales with $\varepsilon^{-2}$, and qualitatively agrees with the behavior observed in the thinnest $\mathrm{NbN}$ films [21].

There are, however, two difficulties: The first is that the zero-dimensional conductivity has to be converted into the measured conductance per square, dividing it by the square of a suitable length scale $\ell$, possibly related with the size of the fluctuating (nano)domains. Thus, one should expect

$$
\delta \sigma_{\text {measured }}=\left(\frac{\xi_{0}}{\ell}\right)^{2} \frac{\pi e^{2}}{4 \hbar \varepsilon^{2}} .
$$

The second, more fundamental, difficulty is to provide a clear meaning to the formal limit $D \rightarrow 0$ of the AL paraconductivity. Although the supergrains may behave as quantum dots, in the end, the system is two dimensional, the supergrains are eventually coupled, and the paraconductive fluctuations must cross over to $D=2$. The formal limit does not allow for a description of this crossover and the identification of the relevant length scales. The next appendices are devoted to the attempt to describe the crossover.

\section{APPENDIX B: EFFECTIVE DENSITY OF STATES}

A crucial remark of Ref. [29] is that the AL theory can be cast in an even more general form, introducing a suitable weighted density of states

$$
\mathcal{N}_{D}(v) \equiv \int \frac{d^{D} \mathbf{q}}{(2 \pi)^{D}}\left(\nabla_{\mathbf{q}} v_{\mathbf{q}}\right)^{2} \delta\left(v-v_{\mathbf{q}}\right),
$$

which allows to write the paraconductivity in the form

$$
\delta \sigma_{D}=\frac{\pi e^{2}}{4 \hbar D} \int_{0}^{\infty} d v \frac{\mathcal{N}_{D}(v)}{(\varepsilon+v)^{3}} .
$$

The standard AL result is recovered with

$$
\begin{aligned}
\mathcal{N}_{D}(v) & \equiv \frac{4 \xi_{0}^{4} \Omega_{D}}{(2 \pi)^{D}} \int_{0}^{\infty} d q q^{D+1} \delta\left(v-\xi_{0}^{2} q^{2}\right) \\
& =\frac{2 \xi_{0}^{2-D} \Omega_{D}}{(2 \pi)^{D}} \int_{0}^{\infty} d z z^{D / 2} \delta(v-z) \\
& =\frac{2 \xi_{0}^{2-D} \Omega_{D}}{(2 \pi)^{D}} v^{D / 2},
\end{aligned}
$$

yielding

$$
\delta \sigma_{D}=\frac{\pi e^{2} \xi_{0}^{2-D} \Omega_{D}}{2 \hbar D(2 \pi)^{D}} \int_{0}^{\infty} d v \frac{v^{D / 2}}{(\varepsilon+v)^{3}} .
$$

However, we shall argue that the observed 0D-2D crossover stems from a different form of $\mathcal{N}_{D}(v)$, that results from the supergrains (endowed with an internal structure) being connected in a $2 \mathrm{D}$ network.

Let us set $D=2$ for the sake of definiteness, assuming a two-dimensional system. Then, $\mathcal{N}_{D=2}(\nu)=\nu / \pi$ and the 
standard AL expression is cast in the form

$$
\delta \sigma_{D=2}=\frac{e^{2}}{8 \hbar} \int_{0}^{\infty} d v \frac{v}{(\varepsilon+v)^{3}}=\frac{e^{2}}{16 \hbar \varepsilon} .
$$

This result crucially depends on the presence of the factor $v$ in the numerator of the integrand. Now, we argue that this $v$ should be replaced by a crossover function

$$
g(v)= \begin{cases}v, & \text { for } v \leqslant \bar{v} \\ \bar{v}, & \text { for } v>\bar{v}\end{cases}
$$

where $\bar{v}$ is a suitable constant, the connection with the weighted density of states being established as $\mathcal{N}_{D=2}(\nu)=$ $g(v) / \pi$, and show that this expression produces a $0 \mathrm{D}-2 \mathrm{D}$ crossover at $\varepsilon \approx \bar{\nu}$.

The expression

$$
\begin{aligned}
\delta \sigma & =\frac{e^{2}}{8 \hbar} \int_{0}^{\infty} d v \frac{g(v)}{(\varepsilon+v)^{3}} \\
& =\frac{e^{2}}{8 \hbar}\left[\int_{0}^{\bar{v}} d v \frac{v}{(\varepsilon+v)^{3}}+\bar{v} \int_{\bar{v}}^{\infty} d v \frac{1}{(\varepsilon+v)^{3}}\right]
\end{aligned}
$$

is readily calculated and gives

$$
\delta \sigma=\frac{e^{2}}{16 \hbar} \frac{\bar{v}}{\varepsilon(\varepsilon+\bar{v})} .
$$

For $\varepsilon \ll \bar{\nu}$, the standard 2D AL behavior is recovered, whereas for $\varepsilon \gg \bar{v}$ a OD behavior is found:

$$
\delta \sigma \approx \frac{e^{2} \bar{v}}{16 \hbar \varepsilon^{2}} .
$$

A rough estimate gives $\varepsilon \approx \xi_{0}^{2} / \xi^{2}$, where $\xi$ is the temperature-dependent correlation length that diverges at the transition. Then, the 0D-2D crossover takes place at a temperature $\bar{T}$ such that $\xi(\bar{T}) \approx \xi_{0} \sqrt{\bar{\nu}}$. A comparison with the formal OD limit of the AL theory allows us to identify the scale $\bar{v}$ as

$$
\bar{v} \approx 4 \pi\left(\frac{\xi_{0}}{\ell}\right)^{2}
$$

and to extract the typical size of the supergrains

$$
\ell \approx \sqrt{\frac{4 \pi}{\bar{v}}} \xi_{0} .
$$

But for trivial numerical prefactors, we assume that $\ell$ can be identified with the scale $L_{i}$ in the main text. The question then arises on the origin and meaning of the crossover function $g(v)$. In the next Appendix, we shall develop a phenomenological dispersion model for collective paraconductive fluctuations. Hereafter, the expression $v_{q}$ will be called dispersion law, although it is more precisely a wave-vector-dependent contribution to the inverse lifetime of the fluctuations, which have a diffusive character at long wavelengths.

\section{APPENDIX C: PHENOMENOLOGICAL DISPERSION MODEL}

Let us adopt an isotropic model with

$$
v_{q}= \begin{cases}\bar{v}\left(\frac{q}{\bar{q}}\right)^{2}, & \text { for } q \leqslant \bar{q} \\ \bar{v}\left[1+\log \left(\frac{q}{\bar{q}}\right)^{2}\right], & \text { for } q>\bar{q}\end{cases}
$$

where $\bar{q}$ is a characteristic wave-number scale. This model dispersion behaves as $q^{2}$ at small $q$ (yielding diffusive longwavelength, i.e., hydrodynamic, paraconductive fluctuations), but changes concavity and flattens for $q>\bar{q}$. Note that $v_{q}$ and its first derivative are continuous. The weighted density of states for this model in two dimensions is

$$
\begin{aligned}
& \mathcal{N}_{D=2}(v) \\
& =\left\{\begin{array}{l}
\frac{1}{2 \pi} \int_{0}^{\bar{q}} d q q\left(\frac{2 \bar{v}}{\bar{q}^{2}} q\right)^{2} \delta\left(v-\frac{\bar{v}}{\bar{q}^{2}} q^{2}\right)=\frac{1}{\pi} v, \\
\text { for } v \leqslant \bar{v} \\
\frac{1}{2 \pi} \int_{\bar{q}}^{\infty} d q q\left(\frac{2 \bar{v}}{q}\right)^{2} \delta\left(v-\bar{v}\left[1+\log \left(\frac{q}{\bar{q}}\right)^{2}\right]\right)=\frac{1}{\pi} \bar{v}, \\
\quad \text { for } v>\bar{v}
\end{array}\right.
\end{aligned}
$$

and reproduces, except for a trivial prefactor, the crossover function $g(v)$ that yields a 0D-2D crossover in the AL paraconductivity. Taking $\bar{v} \equiv \xi_{0}^{2} \bar{q}^{2}$, to recover the standard expression at small $q$, we can identify $\bar{q} \approx \sqrt{4 \pi} / \ell$. So, we can interpret our result in the following manner. For $q<\bar{q}$, the long-wavelength physics dominates and the paraconductive fluctuations are diffusive within a homogeneous (in the average) two-dimensional system. For $q>\bar{q}$, the intrasupergrain physics becomes important and the characteristic timescale increases with respect to pure diffusion.

An alternative dispersion law is

$$
v_{q}=\bar{v} \log \left[1+\left(\frac{q}{\bar{q}}\right)^{2}\right],
$$

which again recovers the diffusive behavior $\propto q^{2}$ at small $q$ and is logarithmic at large $q$. This expression is adopted in the main text for definiteness because it has the advantage of being a single expression rather than being defined piecewise. In this case, the weighted density of states

$$
\mathcal{N}_{D=2}(v)=\frac{1}{\pi} \bar{v}\left(1-\mathrm{e}^{-v / \bar{v}}\right)
$$

is, again up to a prefactor, a smoothened version of the crossover function $g(v)$ that yields a 0D-2D crossover in the AL paraconductivity. We notice that the asymptotic behaviors for $v \gg \bar{v}$ or $v \ll \bar{v}$ are relevant to fit the the 0D-2D crossover observed in the paraconductivity data [21], while the intermediate behavior, as customary in crossover phenomena, is much less constrained by experiments.
[1] A. M. Goldman and N. Marković, Superconductor-insulator transitions in the two-dimensional limit, Phys. Today 51(11), 39 (1998).

[2] G. N. Gol'tsman et al., Picosecond superconducting singlephoton optical detector, Appl. Phys. Lett. 79, 705 (2001).
[3] M. Hofherr, D. Rall, K. Ilin, M. Siegel, A. Semenov, H. W. Hübers, and N. A. Gippius, Intrinsic detection efficiency of superconducting nanowire single-photon detectors with different thicknesses, J. Appl. Phys. 108, 014507 (2010). 
[4] Y. Dubi, Y. Meir, and Y. Avishai, Nature of the superconductorinsulator transition in disordered superconductors, Nature (London) 449, 876 (2007).

[5] M. Bard, I. V. Protopopov, I. V. Gornyi, A. Shnirman, and A. D. Mirlin, Superconductor-insulator transition in disordered Josephson-junction chains, Phys. Rev. B 96, 064514 (2017).

[6] B. Sacépé, C. Chapelier, T. I. Baturina, V. M. Vinokur, M. R. Baklanov, and M. Sanquer, Disorder-Induced Inhomogeneities of the Superconducting State Close to the SuperconductorInsulator Transition, Phys. Rev. Lett. 101, 157006 (2008).

[7] A. M. Finkelstein, Superconductivity-transition temperature in amorphous films, Pis'ma Zh. Eksp. Teor. Fiz. 45, 37 (1987) [JETP Lett. 45, 46 (1987)].

[8] I. V. Lerner, A. A. Varlamov, and V. M. Vinokur, Fluctuation Spectroscopy of Granularity in Superconducting Structures, Phys. Rev. Lett. 100, 117003 (2008).

[9] G. M. Klemencic, J. M. Fellows, J. M. Werrell, S. Mandal, S. R. Giblin, R. A. Smith, and O. A. Williams, Fluctuation spectroscopy as a probe of granular superconducting diamond films, Phys. Rev. Mater. 1, 044801 (2017).

[10] M. P. A. Fisher, G. Grinstein, and S. M. Girvin, Presence of Quantum Diffusion in Two Dimensions: Universal Resistance at the Superconductor-Insulator Transition, Phys. Rev. Lett. 64, 587 (1990).

[11] M. P. A. Fisher, Quantum Phase Transitions in Disordered TwoDimensional Superconductors, Phys. Rev. Lett. 65, 923 (1990).

[12] G. Seibold, L. Benfatto, C. Castellani, and J. Lorenzana, Superfluid Density and Phase Relaxation in Superconductors with Strong Disorder, Phys. Rev. Lett. 108, 207004 (2012).

[13] M. Feigel'man, L. Ioffe, V. Kravtsov, and E. Yuzbashyan, Eigenfunction Fractality and Pseudogap State near the Superconductor-Insulator Transition, Phys. Rev. Lett. 98, 027001 (2007).

[14] M. V. Feigel'man, L. B. Ioffe, V. E. Kravtsov, and E. Cuevas, Fractal superconductivity near localization threshold, Ann. Phys. (NY) 325, 1390 (2010).

[15] L. B. Ioffe and M. Mézard, Disorder-Driven Quantum Phase Transitions in Superconductors and Magnets, Phys. Rev. Lett. 105, 037001 (2010).

[16] G. Lemarié, A. Kamlapure, D. Bucheli, L. Benfatto, J. Lorenzana, G. Seibold, S. C. Ganguli, P. Raychaudhuri, and C. Castellani, Universal scaling of the order-parameter distribution in strongly disordered superconductors, Phys. Rev. B 87, 184509 (2013).

[17] B. Sacépé, T. Dubouchet, C. Chapelier, M. Sanquer, M. Ovadia, D. Shahar, M. Feigel'man, and L. Ioffe, Localization of preformed Cooper pairs in disordered superconductors, Nat. Phys. 7, 239 (2011).

[18] K. Bouadim, Y. L. Loh, M. Randeria, and N. Trivedi, Single- and two-particle energy gaps across the disorder-driven superconductor-insulator transition, Nat. Phys. 7, 884 (2011).

[19] N. Trivedi, Y. L. Loh, K. Bouadim, and M. Randeria, Emergent granularity and pseudogap near the superconductor-insulator transition, J. Phys.: Conf. Ser. 376, 012001 (2012).

[20] A. Kamlapure, T. Das, S. C. Ganguli, J. B. Parmar, S. Bhattacharyya, and P. Raychaudhuri, Emergence of nanoscale inhomogeneity in the superconducting state of a homogeneously disordered conventional superconductor, Sci. Rep. 3, 2979 (2013).

[21] C. Carbillet, S. Caprara, M. Grilli, C. Brun, T. Cren, F. Debontridder, B. Vignolle, W. Tabis, D. Demaille, L. Largeau, K. Ilin, M. Siegel, D. Roditchev, and B. Leridon, Confinement of superconducting fluctuations due to emergent electronic inhomogeneities, Phys. Rev. B 93, 144509 (2016).

[22] A. Larkin and A. Varlamov, Theory of Fluctuations in Superconductors (Oxford University Press, Oxford, 2005).

[23] A. A. Varlamov, A. Galda, and A. Glatz, Fluctuation spectroscopy: From Rayleigh-Jeans waves to Abrikosov vortex clusters, Rev. Mod. Phys. 90, 015009 (2018).

[24] S. Caprara, M. Grilli, B. Leridon, and J. Vanacken, Paraconductivity in layered cuprates behaves as if due to pairing of nearly free quasiparticles, Phys. Rev. B 79, 024506 (2009).

[25] E. Abrahams, M. Redi, and J. W. F. Woo, Effect of fluctuations on electronic properties above the superconducting transition, Phys. Rev. B 1, 208 (1970).

[26] R. W. Cohen, B. Abeles, and C. R. Fuselier, Effect of Fluctuations in the Superconducting Order Parameter on the Tunneling Density of States, Phys. Rev. Lett. 23, 377 (1969).

[27] C. Di Castro, R. Raimondi, C. Castellani, and A. A. Varlamov, Superconductive fluctuations in the density of states and tunneling resistance in high- $T_{c}$ superconductors, Phys. Rev. B 42, 10211 (1990).

[28] In a theoretical language, we remind that the self-interaction of fluctuating Cooper pairs reduce the critical temperature of the noninteracting (Gaussian) theory. Fixing a given infrared cutoff, one obtains a larger suppression in 0D than in 2D. Therefore, as long as the system is observed in a regime where the fluctuations have a OD character, the system "sees" a somewhat lower critical temperature.

[29] S. Caprara, M. Grilli, B. Leridon, and J. Lesueur, Extended paraconductivity regime in underdoped cuprates, Phys. Rev. B 72, 104509 (2005). 